\title{
Distal-to-proximal joint kinetics redistribution is a main contributor to reduced walking economy in older adults
}

\begin{tabular}{|r|l|}
\hline Journal: & Scandinavian Journal of Medicine and Science in Sports \\
\hline Manuscript ID & SJMSS-O-1170-20 \\
\hline Manuscript Type: & Original Article \\
\hline Author: & 07-Oct-2020 \\
\hline Complete List of Authors: & $\begin{array}{l}\text { Delabastita, Tijs; KU Leuven, Human Movement Biomechanics Research } \\
\text { Group, Department of Movement Sciences } \\
\text { Hollville, Enzo; KU Leuven, Human Movement Biomechanics Research } \\
\text { Group, Department of Movement Sciences } \\
\text { Catteau, Andreas; KU Leuven, Human Movement Biomechanics Research } \\
\text { Group, Department of Movement Sciences } \\
\text { Cortvriendt, Philip; KU Leuven, Human Movement Biomechanics } \\
\text { Research Group, Department of Movement Sciences } \\
\text { De Groote, Friedl; KU Leuven, Human Movement Biomechanics Research } \\
\text { Group, Department of Movement Sciences } \\
\text { Vanwanseele, Benedicte; KU Leuven, Human Movement Biomechanics } \\
\text { Research Group, Department of Movement Sciences }\end{array}$ \\
\hline Keywords: & ultrasound, gait, energy cost, muscle-tendon, elderly, joint power \\
\hline & \\
\hline
\end{tabular}

\section{SCHOLARONE Manuscripts}


$1 \quad \underline{\text { Title }}$

2 Distal-to-proximal joint kinetics redistribution is a main contributor to reduced walking

3 economy in older adults

4

$5 \quad$ Running head

$6 \quad$ Hip role in reduced elderly walking economy

$7 \quad$ Authors

8 Tijs Delabastita, Enzo Hollville, Andreas Catteau, Philip Cortvriendt, Friedl De Groote,

$9 \quad$ Benedicte Vanwanseele

$10 \underline{\text { Affiliation }}$

11 Human Movement Biomechanics Research Group, Department of Movement Science, KU

12 Leuven, Belgium

13 Corresponding author

14 Benedicte Vanwanseele

15 benedicte.vanwanseele@,kuleuven.be

16 Tervuursevest 101 - bus 1501, 3001 Leuven, Belgium

18 Acknowledgments

19 We thank W. Swinnen, B. Scheepers, and T. Wijgaerts for their help during experimental data 20 collection. 


\section{Abstract \\ 22 \\ Background}

23

24

25

26

27

Age-related neural and musculoskeletal declines affect mobility and the quality of life of old people. To date, the mechanisms underlying the reduced walking economy in older adults still remain elusive. In this study, we wanted to investigate which biomechanical factors were associated with the higher energy cost of walking in older compared to young adults.

Methods

Fourteen young $(24 \pm 2$ years) and older ( $74 \pm 4$ years) adults were tested. Plantarflexor strength and Achilles tendon stiffness were evaluated during a dynamometer test. Medial gastrocnemius fascicle length, ground reaction forces, joint kinematics and oxygen consumption were measured during walking treadmill at $0.83 \mathrm{~m} \cdot \mathrm{s}^{-1}$ and $1.39 \mathrm{~m} . \mathrm{s}^{-1}$. Energy cost of walking, lower-limb joint kinetics, muscle-tendon unit and tendinous tissues length were calculated.

Results

Energy cost of walking was higher at $0.83 \mathrm{~m} \cdot \mathrm{s}^{-1}(+16 \% ; \mathrm{p}=0.005)$ and plantarflexor strength lower $(-31 \% ; p=0.007)$ in older adults. Achilles tendon stiffness $(p=0.66)$ and medial gastrocnemius fascicle length changes did not differ between young and older adults. Ankle kinetics reduction was compensated by hip kinetics increase in older adults during walking. The hip extensor moment was the only significant predictor of the energy cost of walking.

\section{Conclusion}

The higher energy cost of walking in older adults is mainly associated with the distal-toproximal redistribution of joint kinetics in older adults during walking possibly due to 
43 plantarflexor weakness. In our study, medial gastrocnemius fascicle and tendinous tissue

44 behavior did not explain the higher energy cost of walking between young and older adults.

\section{Keywords}

46 ultrasound, gait, energy cost, muscle-tendon, elderly, joint power 


\section{INTRODUCTION}

Slow preferred walking speed is a predictor of mobility limitations and hospitalization ${ }^{1}$ and is related to the high energy cost of walking in older adults. Specifically, a rise in energetic cost can predict the rate of preferred walking speed decline after the age of 65 years ${ }^{2}$. It has therefore been suggested that older adults compensated for their poor walking economy by adopting a preferred slow walking speed ${ }^{2}$. However, the biomechanical factors associating with such poor walking economy remain equivocal. A better understanding of these factors may help designing tailored training interventions to improve walking economy and performance in older adults.

Reduced walking economy and impaired mobility in older adults result from a broad range of age-related neural and musculoskeletal changes ${ }^{3-5}$. As a result, older adults have marked deficits in push-off intensity or propulsive power during walking ${ }^{6}$. In addition, ankle plantarflexor moments during push-off are lower and hip extensor moments during mid-stance are higher during walking in older than in young adults ${ }^{7,8}$. This compensatory mechanism is well-known as distal-to-proximal redistribution of positive joint work and power within the lower limb to generate propulsive power during walking 9,10. Previously, using a double inverted pendulum model, it was found that during walking, lower push-off forces at the ground contact point of the trailing leg and higher compensatory hip extensor moments at the leading leg increased the energy dissipation when redirecting the center of mass velocity ${ }^{11}$. This might partly explain why the proximal shift in joint moments would be related to higher whole-body energy cost of walking in older adults ${ }^{12}$. Although lower plantarflexor moments functionally impair older adults' push-off intensity ${ }^{13}$, it yet remains unclear if they are also related to the energy cost of walking.

The age-related deficits in propulsive power during walking is associated with hallmark agerelated reductions in ankle plantarflexor muscle power and work (for review, see ${ }^{6}$ ). In addition, Achilles tendon behavior and properties are altered with ageing ${ }^{14,15}$ and may be another 
72 explanation for the higher energy cost of walking observed in older compared to young adults. Indeed during walking, the Achilles tendon acts as a spring enabling the triceps surae to efficiently produce force near optimal length (i.e., quasi-isometrically) and at very low contraction velocity ${ }^{16}$. Any impairment in Achilles tendon stiffness may in turn affect power generation by altering the operating lengths and contraction velocities of the triceps surae. Older adults generally exhibit lower Achilles tendon stiffness (for review, see ${ }^{17}$ ) which may increase Achilles tendon elongation during walking compared to young adults ${ }^{18}$. Th is might force the triceps surae to operate at shorter length and high shortening velocity during the stance phase of walking ${ }^{18-20}$, thus increasing the generating cost of doing work and likely the whole-body metabolic cost of walking ${ }^{21}$. Very recently, Conway and Franz (2020) observed that medial gastrocnemius fascicles were shorter during walking at preferred and fast walking speed in older adults, which was associated with worse capacity to enhance push-off intensity ${ }^{19}$. These authors also observed slower fascicle rening contraction velocity in older adults whatever the walking speed. Besides, Stenroth et al. (2017) have suggested that older adults adopted a slow preferred walking speed which improves their triceps surae force generation ability, mainly because of plantarflexor weakness and the favorable force-velocity potentials ${ }^{20}$. Although simulation studies already showed that Achilles tendon stiffness influences the energy cost of walking ${ }^{21}$, direct experimental evidence is lacking regarding the relationship between Achilles tendon stiffness, triceps surae behavior and the energy cost of walking in older adults.

The main aim of this study was to investigate which biomechanical factors were associated to the higher energy cost of walking in older compared to young adults at slow and fast walking speed. We first evaluated how young and older adults differ in terms of Achilles tendon stiffness, maximal plantarflexor strength, energy cost of walking, walking capacity, medial gastrocnemius fascicle, muscle-tendon unit and tendinous tissue behavior, and joint kinematics and kinetics. We then used the same variables to predict the energy cost of walking in young 
and older adults. We hypothesized that energy cost of walking, hip extensor and flexor moments and medial gastrocnemius fascicle shortening velocity during walking would be higher in older than in young adults. Moreover, we expected to find lower Achilles tendon stiffness, medial gastrocnemius operating length, maximal plantarflexor strength, ankle plantarflexor moments during walking, and lower maximal walking capacity in older than in young adults. We also expected to find an association between the higher energy cost of walking seen in older adults and lower Achilles tendon stiffness, unfavorable medial gastrocnemius operating length and velocity, lower maximal plantarflexor strength and larger hip kinetics during walking.

\section{METHODS}

\section{Participants}

We determined the sample size of this study using a priori statistical power estimations. The overall effect size of the difference in Achilles tendon stiffness between young and older adults, reported in a systematic review was $1.2{ }^{17}$. The effect size of the difference in maximal ankle plantarflexor moment during walking and the energy cost of walking between young and older adults reported in previous studies was 1.2 (young $136 \pm 27$ N.m ${ }^{-1}$, older $\left.102 \pm 29 \mathrm{~N}^{-m^{-1}}\right)^{9}$ and 1.3 (young $0.179 \pm 0.02 \mathrm{ml} \mathrm{O}_{2} \cdot \mathrm{kg} \cdot \mathrm{m}^{-1}$, older $0.205 \pm 0.02 \mathrm{ml} \mathrm{O}_{2} \cdot \mathrm{kg} \cdot \mathrm{m}^{-1}$ ) ${ }^{22}$, respectively. With $\alpha$-error probability of 0.05 and statistical power of 0.8 , a sample size of 13 young and older subjects would be sufficient to detect age-related differences in Achilles tendon stiffness, maximal ankle plantarflexor moment during walking and in the energy cost of walking using two-tailed Mann-Whitney U tests.

When piloting the study, we noticed that the ultrasound image quality was worse in older than in young adults. To account for exclusion of older subjects due to poor image quality, we recruited 16 young adults (age: $23 \pm 2$ years; body mass: $63 \pm 12 \mathrm{~kg}$; length: $165 \pm 7 \mathrm{~cm}$; 8 males, 8 females) and 18 older adults (age: $75 \pm 4$ years; body mass: $73 \pm 12 \mathrm{~kg}$; length: $170 \pm$ 
$1219 \mathrm{~cm}$; 9 males, 9 females). We included healthy young (between 20 and 30 years) or older

122 (between 68 and 85 years old) subjects that did not participate in competitive sports and did not 123 perform structured exercise more than twice a week over the last three months. This study was 124 approved by the Medical Ethics Committee UZ/KU Leuven. The subjects signed written 125 informed consent before participating in the study in accordance with the declaration of 126 Helsinki.

\section{Experimental design}

128 We tested the participants during a single session comprising three tests: a) an overground 129 walking test, where we quantified maximal walking capacity, b) a treadmill walking test, where 130 we collected data to quantify medial gastrocnemius fascicle length and contraction velocity, 131 gait kinematics and kinetics and the energy cost of walking, and c) a dynamometer test, where 132 we collected data to quantify Achilles tendon stiffness and maximal plantarflexor strength.

\section{Overground walking}

134 To quantify maximal walking capacity, we measured the distance covered during a six-minute 135 walk test, i.e. the six-minute walk distance, with participants being barefoot ${ }^{23}$.

\section{Treadmill walking}

137 Actual testing only started after an extensive familiarization of at least 20 minutes to the 138 instrumented treadmill (M-Gait, Motekforce Link, Amsterdam, The Netherlands) ${ }^{24}$. During 139 testing, the subjects walked barefoot at $0.83 \mathrm{~m} \cdot \mathrm{s}^{-1}\left(3 \mathrm{~km} \cdot \mathrm{h}^{-1}\right)$ and at $1.39 \mathrm{~m} \cdot \mathrm{s}^{-1}\left(5 \mathrm{~km} \cdot \mathrm{h}^{-1}\right)$ for 5 140 to 8 minutes. Between the familiarization, and between the testing trials, the subjects could rest 141 until they sufficiently recovered. The participants walked barefoot to enable precise foot marker 142 placement and to avoid inter-subject variability due to shoe and insole type. 
144 To quantify the energy cost of walking, we continuously measured oxygen consumption and 145 carbon dioxide production (Oxycon Mobile Pro, Carefusion, Houten, The Netherlands). We 146 only proceeded to the next trial after at least 5 minutes and if the subjects attained a steady state 147 oxygen uptake and carbon dioxide production for at least 2 minutes ${ }^{22}$. We asked the subjects 148 to maintain their normal dietary pattern and to avoid intense physical activities during 24 hours 149 before the start of the measurement. The subjects did not eat or drink for 2 hours before starting 150 the static standing gas analysis that was performed before treadmill walking.

151 We averaged raw oxygen uptake during the steady state phase in the last two minutes of walking 152 at $0.83 \mathrm{~m} \cdot \mathrm{s}^{-1}$ and $1.39 \mathrm{~m} \cdot \mathrm{s}^{-1}$ (Oxycon Mobile Pro software, Carefusion, Houten, The 153 Netherlands). We subtracted the oxygen uptake during static standing from the oxygen uptake 154 during walking to obtain net oxygen uptake. We normalized this value to body weight and to 155 distance covered to obtain the normalized net oxygen uptake during walking ${ }^{22}$. Normalized net 156 oxygen uptake is a good predictor for energy cost of walking in young and older adults ${ }^{22}$. Lower-limb joint kinematics and kinetics

158 Motion capture data and ground reaction forces were collected during three full strides of 159 walking after 5 to 8 minutes at $0.83 \mathrm{~m} \cdot \mathrm{s}^{-1}$ and at $1.39 \mathrm{~m} \cdot \mathrm{s}^{-1}$. We captured the trajectories of 68 160 reflective markers using thirteen infrared cameras (Vicon, Oxford, UK) recording at $100 \mathrm{~Hz}$. 161 We used an extended full body plug in gait marker set ${ }^{25}$. We measured ground reaction forces 162 using the two built-in force plates in the treadmill at $1000 \mathrm{~Hz}$.

163 We scaled an OpenSim's gait 2392-model to the subject's anthropometry using the marker data 164 collected during a static standing trial ${ }^{26}$. Afterwards, we calculated joint kinematics with a 165 Kalman smoothing algorithm ${ }^{27}$ using the marker data collected during walking. We used the 166 kinematics and ground reaction forces in OpenSim's inverse dynamics tool to calculate joint 167 moments. Next, joint power and joint work were computed. Ankle and hip joint power were 
calculated as the product of the joint moment and the joint angular velocity (derivative of joint angular displacement). Joint work was calculated by the integration of joint power using the trapezium method. Joint kinetics parameters (i.e., moments, power, work) were normalized to the subject's body weight. Afterwards, we identified the maximal ankle plantarflexor moment, maximal hip extensor moment and maximal hip flexor moment per stride before averaging over 3 strides per participant.

\section{Medial gastrocnemius fascicle length and velocity}

To quantify medial gastrocnemius fascicle length and contraction velocity, we collected twodimensional ultrasound images of the medial gastrocnemius muscle fascicles of the right leg. We used a PC-based ultrasound system (Echoblaster 128, UAB Telemed, Vilnius, Lithuania) to record ultrasound images at $60 \mathrm{~Hz}$ using a flat shaped ultrasound probe (wave frequency 8 $\mathrm{MHz}$, field of view $60 \mathrm{~mm}$ ). We carefully placed the probe in line with the muscle fascicles and perpendicular to the deep aponeurosis of the medial gastrocnemius ${ }^{28}$. The probe placement was similar to the placement used in a previous study ${ }^{29}$. We synchronized motion capture and ultrasound data with an electrical pulse at the start and at the end of ultrasound imaging.

The ultrasound images collected during treadmill walking were processed by tracking three lines representing the superficial aponeurosis, the deep aponeurosis and the orientation of the medial gastrocnemius muscle fascicles with a semi-automated algorithm ${ }^{30}$. We linearly extrapolated these lines and calculated medial gastrocnemius fascicle length as the distance between the two aponeuroses parallel to the muscle fascicles and the pennation angle as the angle between the muscle fascicles and the deep aponeurosis ${ }^{16}$. The first derivative of the medial gastrocnemius fascicle length with respect to time was calculated to determine medial gastrocnemius fascicle contraction velocity. We normalized medial gastrocnemius fascicle length to the average fascicle length measured during the static standing trial. Afterwards, we calculated medial gastrocnemius fascicle length operating range throughout the whole stance 
193 phase and throughout terminal stance and pre-swing (50 to $85 \%$ and 85 to $100 \%$ of the stance 194 phase, respectively). We defined operating range as the difference between minimal and 195 maximal normalized fascicle length throughout the time range of interest ${ }^{20}$. We also calculated 196 medial gastrocnemius fascicle contraction velocity at maximal ankle plantarflexor moment and 197 maximal fascicle contraction velocity throughout terminal stance and pre-swing ${ }^{20}$.

198 Additionally, we calculated medial gastrocnemius tendinous tissue length by subtracting medial 199 gastrocnemius fascicle length multiplied by the cosine of its pennation angle from the medial 200 gastrocnemius muscle-tendon length ${ }^{16}$ calculated using OpenSim's muscle analysis tool. We 201 also normalized medial gastrocnemius tendinous tissue length to its length measured during the 202 static standing trial.

\section{Dynamometer testing session}

204 The participants lay prone on the dynamometer seat with the left foot on the footplate (Biodex 205 System 3, Biodex Medical Systems, Inc., New York, USA). The ankle was positioned in 90 206 flexion (neutral position) with the knee and hip fully extended. The foot was tightly strapped to 207 the footplate. Before each trial, we carefully aligned the mid-point of the line between the 208 medial and the lateral ankle with the dynamometer axis. Participants performed four explosive 209 isometric plantarflexions. Peak ankle torque was averaged across the four trials and used as a measure of plantarflexor strength (34).

211 We captured the trajectories of 10 reflective markers using three mobile infrared cameras 212 (Vicon, Oxford, UK) recording at $100 \mathrm{~Hz}$. The marker set and marker placement were similar 213 as during treadmill walking, but we only used the markers on the lateral side of the left leg ${ }^{25}$.

214 We also collected ultrasound images of the medial gastrocnemius muscle-tendon junction 215 during explosive contractions and passive rotations. A PC-based ultrasound system captured images at $30 \mathrm{~Hz}$ using the same probe, wave frequency and field of view as during treadmill 
217 walking. To visualize the medial gastrocnemius muscle-tendon junction, we placed the probe 218 at the distal end of the medial gastrocnemius muscle belly ${ }^{5}$. We slightly moved the probe to 219 obtain images perpendicular to the aponeuroses. We synchronized motion capture and 220 ultrasound data with an electrical pulse at the start and at the end of ultrasound imaging.

221 We quantified Achilles tendon force by dividing the ankle plantarflexor moment by the Achilles tendon moment arm calculated using a hybrid method combining three-dimensional motion analysis and ultrasound ${ }^{31}$. Briefly, ultrasound was used to measure the perpendicular distance from the surface of the skin to the midline of the Achilles tendon, while motion capture was used to determine the perpendicular distance from the bottom of the probe to the ankle joint center. The difference between these two measures was defined as the Achilles tendon moment arm (31). We calculated Achilles tendon elongation during the explosive contractions by

237 For five subjects, we could only use 3 trials due to technical issues.

\section{Statistical analysis}

239 We used Mann-Whitney U tests to compare young and older adults with respect to the discrete 240 parameters Achilles tendon stiffness, maximal plantarflexor strength, energy cost and sixminute walk distance. We used non-parametric statistical tests throughout the study to account 
242 for non-normally distributed outcomes. We applied Sidak-corrections for multiple comparisons 243 (critical alpha-level at 0.0253 for the comparisons of oxygen uptake and at 0.05 for other 244 comparisons).

245 To compare young and older adults with respect to the time-varying parameters ankle and hip 246 joint kinetics, medial gastrocnemius fascicle length and contraction velocity, tendinous tissue 247 length and muscle-tendon unit length, we used repeated measures ANOVA's with statistical 248 non-parametrical mapping (SNPM) ${ }^{34}$. We applied Sidak-corrections for multiple comparisons 249 (critical alpha-level at 0.0253 for the comparisons of ankle and hip joint moments and at 0.0127 250 for other comparisons). We performed these analyses in Matlab 2015b (The Mathworks, Inc., 251 Natick, USA).

252 Partial correlations using non-parametric Spearman's rank correlations adjusted for step 253 frequency were performed to investigate the relationships between the energy cost of walking 254 and, the Achilles tendon stiffness, maximal plantarflexor strength, joint kinetics, medial 255 gastrocnemius fascicle length, and six-minute walk distance. Data for young and older adults 256 were pooled as we did find similar correlation coefficients between both age groups. We applied 257 Sidak-corrections for multiple correlations. The critical alpha level was set to 0.05 for 258 correlations between Achilles tendon stiffness, maximal isometric plantarflexor force, and six259 minute walk distance. The critical alpha level was set to 0.0253 for all other correlations.

260 Based on non-parametric partial correlations, we additionally performed a multiple regression 261 analysis using the three significant independent variables (i.e., hip extensor moment, Achilles 262 tendon stiffness and plantarflexor strength) as inputs to predict the energy cost of walking. Two 263 independent models were tested corresponding to the two controlled walking speeds used in 264 this study. We used bootstrapping to account for non-normally distributed variables. We 265 checked the assumptions of multicollinearity using tolerance and variance inflation factor and 
266

267

268

269

270

271

272

273

274

275

276

277

278

279

280

281

282

283

284

285

reported no violation of the assumptions. All statistical analyses were performed in SPSS Statistics 20 (IBM Corp., Armonk, USA).

\section{Results}

We excluded four older participants due to problems to perform isometric contractions without compensations (two participants) and insufficient ultrasound image quality (two participants). As gender influences Achilles tendon stiffness ${ }^{5}$, we excluded the last two young male participants included in the study to have the same distribution of males and females in both groups. The results of 14 healthy young adults (age: $24 \pm 2$ years; height $170 \pm 10 \mathrm{~cm}$; body mass $63 \pm 13 \mathrm{~kg} ; 7$ male and 7 female) and 14 healthy older adults (age $74 \pm 4$ years, height $165 \pm 6 \mathrm{~cm}$, weight $73 \pm 12 \mathrm{~kg}, 7$ male and 7 female) are reported.

\section{Young vs. older adults}

Older adults exhibited a higher net energy cost of walking than young adults when walking at $0.83 \mathrm{~m} \cdot \mathrm{s}^{-1}$ (on average $+16 \%$ ) while we observed a trend for higher net energy cost of walking at $1.39 \mathrm{~m} \cdot \mathrm{s}^{-1}$ (on average $+12 \% ; \mathrm{p}=0.044$; alpha level $\leq 0.0253$; Table 1). Moreover, six-minute walk distance was lower in older than in young adults (on average $-17 \%$; Table 1). Maximal plantarflexor strength was also lower in older than in young adults (on average -31\%). However, we observed similar Achilles tendon stiffness between young and older adults $(\mathrm{p}=0.663)$.

No statistical differences were observed in medial gastrocnemius muscle-tendon unit, fascicle and tendinous tissue length changes and velocity between young and older adults during the stance phase of walking at both speeds (all p-values $>0.05$; Fig. 1). We also observed similar fascicle operating length at both slow and fast walking speeds in both populations (Fig. 1C).

There was a distal-to-proximal joint kinetics redistribution during walking at both slow and fast speeds in older adults (Fig. 2-4). Ankle plantarflexor moment during terminal stance propulsion was lower in older than in young adults (-9 and $-14 \%$ at slow and fast speed, respectively; Fig. 
290 2A) while hip extensor moment during loading response and mid-stance was higher in older 291 than in young adults $(+32 \%$ and $+30 \%$ at slow and fast speed, respectively; Fig. 2B). The age292 related differences in hip moments were more pronounced at higher walking speed. When 293 increasing walking speed, hip extensor moment during loading response and mid-stance 294 increased more in older than in young adults (Fig. 2B). Hip flexor moment during pre-swing 295 was also higher in older than in young adults (Fig. 2B). Similarly, we observed larger hip 296 extension power at mid-stance $(+25 \%$ and $+32 \%$ at slow and fast speed, respectively) and hip 297 flexion power at terminal stance $(+31 \%$ and $+24 \%$ at slow and fast speed, respectively) in older 298 adults (Fig. 3B). This compensates for reduced ankle power during propulsion at slow (-20\%) 299 and fast walking speeds (-30\%; Fig. 3A). We also found higher positive hip work and lower 300 positive ankle work in older compared to young adults (Fig. 4).

\section{Partial correlation analysis}

302 Partial non-parametric correlations between the energy cost of walking at $0.83 \mathrm{~m} \cdot \mathrm{s}^{-1}$ and $3031.39 \mathrm{~m} . \mathrm{s}^{-1}$ and the main variables, adjusted for step frequency, are reported in Table 2. Higher 304 energy cost of walking was significantly associated with higher hip extensor moment 305 (r: .38 to .48), lower Achilles tendon stiffness (r: -.57 to -.52), lower plantarflexor strength (r: 306.47 to -.43 ), higher hip positive work (r: .45 to .51 ), and higher hip extension power at mid307 stance (r: .45 to .56).

308 Multiple regression analysis

309 The multiple regression models predicted up to $38 \%$ of the variance in the energy cost of 310 walking (Table 3). The independent variables (i.e., hip extensor moment, Achilles tendon 311 stiffness and ankle plantarflexor strength) statistically predicted the energy cost of walking at 312 both $0.83 \mathrm{~m} \cdot \mathrm{s}^{-1}\left(\mathrm{R}^{2}=0.45\right.$; adjusted $\left.\mathrm{R}^{2}=0.38 ; \mathrm{p}=0.002\right)$ and $1.39 \mathrm{~m} \cdot \mathrm{s}^{-1}\left(\mathrm{R}^{2}=0.42\right.$; adjusted $\left.313 \mathrm{R}^{2}=0.35 ; \mathrm{p}=0.004\right)$. However, only hip extensor moment was independent predictor of the 
energy cost of walking in these models (p-values: 0.012 and 0.040 ; standardized coefficients $\beta$ : 0.562 and 0.381 , respectively at $0.83 \mathrm{~m} \cdot \mathrm{s}^{-1}$ and $\left.1.39 \mathrm{~m} \cdot \mathrm{s}^{-1}\right)$.

\section{Discussion}

In this study, we experimentally compared the walking economy of young and older adults and identified the biomechanical factors that were associated with the energy cost of walking at slow and fast speeds. We observed that older adults had a higher energy cost of walking compared to young adults at matched speed. This was accompanied by lower ankle kinetics (i.e., moment, power and work) during the propulsion phase of walking, mainly compensated by larger hip kinetics in early/mid-stance. In addition, we found that the hip extensor moment, Achilles tendon stiffness and plantarflexor strength were associated with the energy cost of walking. However, only the hip extensor moment was an independent predictor of the energy cost of walking. The current study highlights the major influence of the age-related distal-toproximal redistribution of joint kinetics on the energy cost of walking, with increasing demand placed at the hip joint increasing the energy cost of walking in older adults.

The effect of ageing on walking economy has been studied extensively with consistent findings of higher gross and net cost of walking in healthy older adults compared to their younger counterparts (e.g., for review see ${ }^{35}$ ). In line with previous observations, we also reported a $16 \%$ to $12 \%$ higher net energy cost of walking in older than in young adults at both slow $\left(0.83 \mathrm{~m} . \mathrm{s}^{-}\right.$ $\left.{ }^{1}\right)$ and fast speeds $\left(1.39 \mathrm{~m} \cdot \mathrm{s}^{-1}\right)^{22,35,36}$. This increase in the energy cost of walking in older adults is multifactorial and remains unclear. Previous hypotheses about the role of gait instability ${ }^{22}$ and individual leg work ${ }^{37}$ have failed or partly explain (i.e., antagonist activity ${ }^{38}$ ) this agerelated higher energy cost of walking. Based on our results, we posit that the higher mechanical output from muscles spanning the hip, i.e. extensors during early/mid-stance and flexors during 
338 late stance/early swing, is one of the main contributors to the higher energy cost of walking 339 observed in older adults.

\section{$340 \quad$ Which driven mechanisms?}

341 Prior studies highlighted the key role of neural control/strategies in the age-related increase in 342 the energy cost of walking ${ }^{36,38}$. Specifically, increased antagonist leg muscle coactivation 343 explained around one third of the oxygen consumption increase in older adults ${ }^{36,38}$. We now 344 discuss about other potential driven mechanisms explaining the age-related decreased walking 345 economy in older adults.

Age-related distal-to-proximal redistribution of joint kinetics

347 The higher mechanical output from the hip is mainly caused by the well-known age-related 348 distal-to-proximal redistribution of joint kinetics during walking ${ }^{9,10}$ with a shift strategy in 349 mechanical output from the ankle to the hip in older adults. In addition, it has previously been 350 shown in young adults that an experimental reduction of ankle plantarflexor power during 351 walking was accompanied by a higher reliance to the proximal leg muscles and an increase in 352 the energy cost of walking ${ }^{39}$. In our study, we observed that changes in ankle kinetics were 353 inversely related to hip muscle kinetics with an age-related reduction of 20 to $30 \%$ in ankle 354 power and increase of 25 to $32 \%$ in hip power. We also observed similar magnitude of positive 355 ankle work reduction (-26 to $-27 \%$ ) and hip positive work increase (29 to $30 \%$ ) in older adults 356 during walking at both speeds. However, the age-associated reduction in peak ankle moment 357 during push-off was relatively smaller in comparison to the higher hip extensor moment. 358 Deficits in ankle moment emerged as the lone genuine functionally limiting impairment in older 359 adults ${ }^{13}$. Recently, Conway and Franz demonstrated that ankle moment was the main limiting 360 factor of push-off intensity and thereby walking performance (i.e., walking speed). In our study, 361 we observed lower peak ankle moment in older adults during walking at both slow and fast 
362 speeds but this was not correlated to the energy cost of walking $\left(\mathrm{r}: .067, \mathrm{p}=0.74\right.$ at $0.83 \mathrm{~m} . \mathrm{s}^{-1}$; $363 \mathrm{r}: .266, \mathrm{p}=0.18$ at $\left.1.39 \mathrm{~m} \cdot \mathrm{s}^{-1}\right)$. The overall decrease in ankle kinetics has consequences on 364 propulsive power generation in older adults ${ }^{6}$ and partly arise from lower ankle plantarflexor 365 strength (-31\% in our study), a decrease in plantarflexors force-generating capacity and/or their 366 inability to elicit the use of propulsive reserve capacity during walking ${ }^{6}$. Besides, Achilles sub367 tendons dynamics ${ }^{40}$ and Achilles tendon moment arm ${ }^{41}$ could also have affected peak ankle 368 joint moment, work and power during walking in older adults and may indirectly play a role in 369 the redistribution of joint kinetics towards proximal hip muscles.

370 Using multiple regression analyses, we identified hip extensor moment as the only significant 371 predictive factor of the energy cost of walking, explaining between $35 \%$ and $38 \%$ of the 372 variance in the energy cost of walking (Table 2). Together, these results agree with the majority 373 of previous studies regarding the 'biomechanical plasticity/elasticity' of older adult's gait 374 pattern ${ }^{8,10,38,42}$ and highlights the key role of the hip to compensate decreased ability of older 375 adults to use their ankle musculature during walking. Although experimental evidence is 376 lacking, it has previously been suggested that the hip muscles (long fibers and short tendons with little elastic energy storing capacity) generate muscle forces during walking less efficiently than the triceps surae muscles (short fibers and long tendons with higher elastic energy storing capacity) ${ }^{20}$. In addition, a previous study based on computer simulations already suggested that using the hip extensor muscles during mid-stance instead of the ankle muscles during push-off 381 to redirect the center of mass during walking increases the energy cost of walking ${ }^{43}$. Although, how much leg swing contributes to the metabolic cost of walking is still debated, it is clear that the hip flexors are active during swing and consume metabolic energy ${ }^{44}$. In our study, we found that older adults exhibited larger peak power produced by hip flexors during late stance/preswing compared to young adults which could also contribute to the overall increase in the energy cost of walking with age. More direct evidence should confirm this result in the future. 
387

388

389

390

391

392

393

394

395

396

397

398

399

400

401

402

403

404

405

406

407

408

409

410

411

\section{Influence of tendon properties and fascicle dynamics}

We found a significant negative partial correlation between Achilles tendon stiffness and the energy cost of walking at both speeds (r: -.52 and -.57), with a trend for explaining part of the variance in the energy cost of walking at faster walking speed (i.e., $1.39 \mathrm{~m} . \mathrm{s}^{-1}$ ). However, we did not find age-related differences in Achilles tendon stiffness, which is in contrast with most of previous literature ${ }^{17}$. Therefore, in our study sample, Achilles tendon stiffness was not associated with age-related differences in the energy cost of walking. A selection bias might explain why Achilles tendon stiffness was not different between young and older participants in our study. Possibly, young adults were weaker and/or older adults were stronger in our study than in other studies. For instance, maximal isometric plantarflexor strength was on average $31 \%$ lower in older than in young adults in our study but $39 \%$ lower in a study that showed that Achilles tendon stiffness is lower in older than in young adults ${ }^{5}$.

Furthermore, the similar Achilles tendon stiffness found between young and old adults is consistent with our observations of similar gastrocnemius medialis muscle-tendon interactions during walking in old and young adults. Medial gastrocnemius fascicle length and fascicle contraction velocity did not differ between young and older adults at both slow and fast walking speeds. This result is in agreement with a previous study ${ }^{20}$ at matched speed (i.e., $\sim 1.35 \mathrm{~m} . \mathrm{s}^{-1}$ ) corresponding to the fast walking speed in our study. We further demonstrated that this similar behavior between age groups is also persistent at slower matched walking speed. Due to triceps surae working on the ascending limb of the force-length relationship during walking ${ }^{20,21}$, the shortening of the fibers tends to limit force-generation capacity and it is not surprising to find similar fascicle length changes between age groups. Unlike at matched speed, previous studies showed that older adults improved their triceps surae force-generation ability due to more favorable force-velocity potentials when walking at preferred speed ${ }^{19,20}$. Therefore, despite altered tendon properties or not (e.g., not found in our study), medial gastrocnemius in older 
412 adults can operate relatively efficiently at both matched and preferred walking speeds ${ }^{20}$. While 413 other findings on lateral gastrocnemius ${ }^{18}$ are in line with these results, it seems that there is 414 more room for debate on the role of soleus and the age-related differences in force-generation 415 ability during walking ${ }^{20,45}$. It is possible that unfavorable shifts in soleus force-length and force416 velocity potentials reduce the force-generating capacity in older adults, which may in turn drive 417 older adults to use more hip extensors rather than ankle plantarflexors, and by extension 418 increase the energy cost of walking.

\section{Limitations}

421 This study is not without limitations. Our results are dependent on the use of two-dimensional 422 ultrasound to calculate three-dimensional muscle fascicle lengths and muscle-tendon junction 423 displacements. Therefore, extreme care was taken to align the probe with the muscle fascicles 424 and/or aponeuroses before treadmill and dynamometer testing. Our sample size was limited 425 with a small number of young and older participants $(\mathrm{N}=28)$. To calculate partial correlations, 426 young and older adults were treated as one group based on the fact that changes in oxygen 427 uptake ${ }^{22}$, muscle-tendon function ${ }^{4}$ and six-minute walk distance ${ }^{46}$ were gradual with age. To 428 control for potential influence of age group on partial correlations, we visualized each 429 relationship using scatter plots (see supplementary material). Causality cannot be inferred based 430 on the results from the multiple regression analyses. However, we mainly based our 431 interpretation using experimental findings for which the models confirm the key role of the hip 432 joint in the energy cost of walking. Other study design may help to unravel which mechanisms 433 drive the redistribution of joint kinetics during walking. Such design may be inspired by recent 434 work with assisting/resisting treadmill setup where propulsive forces can be 435 monitored/controlled to affect ankle push-off power ${ }^{13,42}$ while the energy cost of walking could 436 be simultaneously measured. The method used to evaluate Achilles tendon stiffness is 
437 imperfect. Specifically, during isometric contractions on dynamometer, there is a lift of the heel

438 from the footplate which decreases the accuracy of force measurements as well as ankle and 439 muscle-tendon junction kinematics despite appropriate joint angular rotation corrections during 440 processing ${ }^{32}$ (see Methods). Lastly, since our measurements only focused on medial 441 gastrocnemius, our findings cannot be generalized to whole triceps surae. This is especially true 442 considering the influence of ageing on Achilles tendon and sub-tendons dynamics (e.g., more 443 uniform sub-tendons deformation in older adults during walking; ${ }^{14}$ ) and the differences in 444 fascicle dynamics/function between triceps surae muscles ${ }^{18,20,45}$.

\section{Conclusion}

446 In this study, we found a reciprocal interplay between hip and ankle function independent of 447 walking speed with ageing which was associated with the energy cost of walking. We posit that 448 the age-related reduction in older adults walking economy mainly comes from an increase in 449 hip joint contribution to propulsive power during walking. This shift towards proximal leg 450 muscles strategy with age likely comes from distal kinetics impairments (e.g., ankle power) 451 during walking and other age-related neural and musculoskeletal changes (e.g., plantarflexor 452 weakness) without altering medial gastrocnemius fascicle and tendinous tissue interactions. 453 This study offers further evidence for the central role of hip extensors and flexors to the 454 hallmark reduction in walking economy with ageing.

\section{$455 \quad$ Perspectives}

456 Training interventions targeting ankle plantarflexor do not always translate into improved ankle 457 moment and power, and increased push-off intensity. In contrast, a training intervention that 458 effectively target both ankle muscle strength and power, and increase propulsive forces during 459 walking might reduce the energy cost of walking in older adults. Recent work using real-time 460 biofeedback during treadmill walking 13,42,47 combined with traditional resistance and 
2

3

4

5

6

8

11

12

13

14

15

16

17

18

19

20

21

22

23

24

25

26

27

28

29

30

31

32

33

34

35

36

37

38

39

40

41

42

43

44

45

46

47

48

49

50

51

52

53

54

55

56

57

58

59

60

461 plyometric training seems promising in order to restore or improve push-off capacity with

462 ageing (e.g., propulsive reserve capacity; ${ }^{6}$ ).

\section{Conflicts of interest}

464 All authors declare that they have no conflict of interest 
465

466

467

468

469

470

471

472

473

474

475

476

477

478

479

480

481

482

483

484

485

486

487

\section{REFERENCES}

1. Cesari M, Kritchevsky SB, Penninx BWHJ, Nicklas BJ, Simonsick EM, Newman AB, Tylavsky FA, Brach JS, Satterfield S, Bauer DC, Visser M, Rubin SM, Harris TB, Pahor M. Prognostic value of usual gait speed in well-functioning older people Results from the health, aging and body composition study. J Am Geriatr Soc 2005;53:1675-1680.

2. Schrack JA, Zipunnikov V, Simonsick EM, Studenski S, Ferrucci L. Rising energetic cost of walking predicts gait speed decline with aging. Journals Gerontol - Ser A Biol Sci Med Sci 2016;71:947-953.

3. Vandervoort AA. Aging of the human neuromuscular system. Muscle and Nerve $2002 ; 25: 17-25$.

4. Narici M V, Maffulli N, Maganaris CN. Ageing of human muscles and tendons. Disabil Rehabil 2008;30:1548-1554.

5. Stenroth L, Peltonen J, Cronin NJ, Sipila S, Finni T. Age-related differences in Achilles tendon properties and triceps surae muscle architecture in vivo. J Appl Physiol 2012;113:1537-1544.

6. Franz JR. The Age-Associated Reduction in Propulsive Power Generation in Walking. Exerc Sport Sci Rev 2016;

7. Boyer KA, Johnson RT, Banks JJ, Jewell C, Hafer JF. Systematic review and metaanalysis of gait mechanics in young and older adults. Exp Gerontol 2017;95:63-70.

8. Kulmala JP, Korhonen MT, Kuitunen S, Suominen H, Heinonen A, Mikkola A, Avela J. Which muscles compromise human locomotor performance with age? J R Soc Interface 2014;11: 
488 9. DeVita P, Hortobagyi T. Age causes a redistribution of joint torques and powers during 489 gait. J Appl Physiol 2000;88:1804-1811.

490

491

492

493

494

495

496

497

498

499

500

501

502

503

504

505

506

507

508

509

510

10. Waanders JB, Hortobágyi T, Murgia A, Devita P, Franz JR. Advanced Age

Redistributes Positive but Not Negative Leg Joint Work during Walking. Med Sci Sports Exerc 2019;51:615-623.

11. Donelan JM, Kram R, Kuo AD. Mechanical work for step-to-step transitions is a major determinant of the metabolic cost of human walking. J Exp Biol 2002;205:3717-3727.

12. Wert DM, Brach J, Perera S, VanSwearingen JM. Gait biomechanics, spatial and temporal characteristics, and the energy cost of walking in older adults with impaired mobility. Phys Ther 2010;90:977-985.

13. Conway KA, Franz JR. Increasing the propulsive demands of walking to their maximum elucidates functionally limiting impairments in older adult gait. J Aging Phys Act 2020;28:1-8.

14. Franz JR, Thelen DG. Imaging and simulation of Achilles tendon dynamics: Implications for walking performance in the elderly. J Biomech 2016;49:1403-1410.

15. Stenroth L, Sillanpãã E, McPhee JS, Narici M V., Gapeyeva H, Pããsuke M, Barnouin Y, Hogrel JY, Butler-Browne G, Bijlsma A, Meskers CGM, Maier AB, Finni T, Sipilã S. Plantarflexor muscle-tendon properties are associated with mobility in healthy older adults. Journals Gerontol - Ser A Biol Sci Med Sci 2015;

16. Lichtwark GA, Bougoulias K, Wilson AM. Muscle fascicle and series elastic element length changes along the length of the human gastrocnemius during walking and running. J Biomech 2007;40:157-164.

17. Delabastita T, Bogaerts S, Vanwanseele B. Age-Related Changes in Achilles Tendon 
511 Stiffness and Impact on Functional Activities: A Systematic Review and Meta-

$512 \quad$ Analysis. J Aging Phys Act 2020;27:116-127.

513 18. Mian OS, Thom JM, Ardigò LP, Minetti AE, Narici M V. Gastrocnemius muscle514 tendon behaviour during walking in young and older adults. Acta Physiol 2007;

515 19. Conway KA, Franz JR. Shorter gastrocnemius fascicle lengths in older adults associate 516 with worse capacity to enhance push-off intensity in walking. Gait Posture 2020;77:89-94.

518 20. Stenroth L, Sipilä S, Finni T, Cronin NJ. Slower Walking Speed in Older Men 519 Improves Triceps Surae Force Generation Ability. Med Sci Sports Exerc 2017;49:158$520 \quad 166$.

521 21. Lichtwark GA, Wilson AM. Optimal muscle fascicle length and tendon stiffness for 522 maximising gastrocnemius efficiency during human walking and running. J Theor Biol $523 \quad 2008 ; 252: 662-673$.

524 22. Malatesta D, Simar D, Dauvilliers Y, Candau R, Borrani F, Prefaut C, Caillaud C. 525 Energy cost of walking and gait instability in healthy 65- and 80-yr-olds. J Appl $526 \quad$ Physiol 2003;95:2248-2256.

527 23. Enright PL. The Six-Minute Walk Test. Respir Care 2003;48:783-785.

528 24. Meyer C, Killeen T, Easthope CS, Curt A, Bolliger M, Linnebank M, Zörner B, Filli L. $529 \quad$ Familiarization with treadmill walking: How much is enough? Sci Rep 2019;9:1-10.

530 25. Van Rossom S, Smith CR, Zevenbergen L, Thelen DG, Vanwanseele B, Van Assche 531 D, Jonkers I. Knee cartilage thickness, T1 $\rho$ and T2 relaxation time are related to $532 \quad$ articular cartilage loading in healthy adults. PLoS One 2017;12:1-16.

533 26. Delp SL, Anderson FC, Arnold AS, Loan P, Habib A, John CT, Guendelman E, Thelen 
534 DG. OpenSim: Open-source software to create and analyze dynamic simulations of 535 movement. IEEE Trans Biomed Eng 2007;

536 27. De Groote F, De Laet T, Jonkers I, De Schutter J. Kalman smoothing improves the 537 estimation of joint kinematics and kinetics in marker-based human gait analysis. J $538 \quad$ Biomech 2008;

539 28. Bolsterlee B, Gandevia SC, Herbert RD. Ultrasound imaging of the human medial 540 gastrocnemius muscle: How to orient the transducer so that muscle fascicles lie in the $541 \quad$ image plane. J Biomech 2016;49:1002-1008.

542 29. Lichtwark GA, Wilson AM. Interactions between the human gastrocnemius muscle and 543 the Achilles tendon during incline, level and decline locomotion. J Exp Biol 544

545 30. Farris DJ, Lichtwark GA. UltraTrack: Software for semi-automated tracking of muscle 546 fascicles in sequences of B-mode ultrasound images. Comput Methods Programs $547 \quad$ Biomed 2016;

548 31. Manal K, Cowder JD, Buchanan TS. Subject-specific measures of achilles tendon 549 moment arm using ultrasound and video-based motion capture. Physiol Rep 2013;1:1$550 \quad 8$.

551 32. Arampatzis A, Monte G De, Karamanidis K. Effect of joint rotation correction when 552 measuring elongation of the gastrocnemius medialis tendon and aponeurosis. $\mathrm{J}$ $553 \quad$ Electromyogr Kinesiol 2008;

554 33. Werkhausen A, Cronin NJ, Albracht K, Paulsen G, Larsen A V., Bojsen-Møller J, 555 Seynnes OR. Training-induced increase in Achilles tendon stiffness affects tendon $556 \quad$ strain pattern during running. PeerJ 2019;2019:. 
557 34. Pataky TC, Vanrenterghem J, Robinson MA. Zero- vs. one-dimensional, parametric vs.

558

559

560

561

562

563

564

565

566

567

568

569

570

571

572

573

574

575

576

577

578

579 non-parametric, and confidence interval vs. hypothesis testing procedures in onedimensional biomechanical trajectory analysis. J Biomech 2015;48:1277-1285.

35. Das Gupta S, Bobbert MF, Kistemaker DA. The Metabolic Cost of Walking in healthy young and older adults - A Systematic Review and Meta Analysis. Sci Rep 2019;9:110.

36. Ortega JD, Farley CT. Effects of aging on mechanical efficiency and muscle activation during level and uphill walking. J Electromyogr Kinesiol 2015;

37. Ortega JD, Farley CT. Individual limb work does not explain the greater metabolic cost of walking in elderly adults. J Appl Physiol 2007;102:2266-2273.

38. Hortobágyi T, Finch A, Solnik S, Rider P, De Vita P. Association between muscle activation and metabolic cost of walking in young and old adults. Journals Gerontol Ser A Biol Sci Med Sci 2011;

39. Huang T wei P, Shorter KA, Adamczyk PG, Kuo AD. Mechanical and energetic consequences of reduced ankle plantar-flexion in human walking. 2015;3541-3550.

40. Clark WH, Franz JR. Triceps surae muscle-subtendon interaction differs between young and older adults. Connect Tissue Res 2020;61:104-113.

41. Rasske K, Thelen DG, Franz JR. Variation in the human Achilles tendon moment arm during walking. Comput Methods Biomech Biomed Engin 2017;20:201-205.

42. Browne MG, Franz JR. More push from your push-off: Joint-level modifications to modulate propulsive forces in old age. PLoS One 2018;13:1-14.

43. Kuo AD. Energetics of actively powered locomotion using the simplest walking model. J Biomech Eng 2002;124:113-120. 
580 44. Umberger BR. Stance and swing phase costs in human walking. J R Soc Interface $581 \quad 2010 ; 7: 1329-1340$.

582 45. Panizzolo FA, Green DJ, Lloyd DG, Maiorana AJ, Rubenson J. Soleus fascicle length 583 changes are conserved between young and old adults at their preferred walking speed. $584 \quad$ Gait Posture 2013;38:764-769.

585 46. Enright PL, Sherrill DL. Reference Equations for the Six-Minute Walk.pdf. Am J 586 Respir Crit Care Med 1998;158:1384-1387.

587 47. Conway KA, Crudup KL, Lewek MD, Franz JR. Effects of Horizontal Impeding Force 588 Gait Training on Older Adult Push-Off Intensity. Med Sci Sport Exerc 2020;Publish $589 \quad$ Ah: 
$\underline{\text { Tables }}$

\begin{tabular}{lcccc}
\hline Parameters & Young adults & Older adults & P-value & $\alpha_{\mathrm{SID}}$ \\
\hline Achilles tendon stiffness $\left(\mathrm{N} . \mathrm{mm}^{-1}\right)$ & $233 \pm 92$ & $219 \pm 108$ & 0.663 & 0.05 \\
Maximal plantarflexor strength (N) & $2051 \pm 531$ & $1406 \pm 299$ & $\mathbf{0 . 0 0 7}$ & 0.05 \\
$\begin{array}{l}\text { Energy cost of walking } \\
\text { at } 0.83 \mathrm{~m}^{-1} \mathrm{~s}^{-1}\left(\mathrm{mlO}_{2} \cdot \mathrm{kg}^{-1} \cdot \mathrm{m}^{-1}\right)\end{array}$ & $0.108 \pm 0.02$ & $0.128 \pm 0.02$ & $\mathbf{0 . 0 0 5}$ & 0.023 \\
$\begin{array}{l}\text { Energy cost of walking } \\
\text { at } 1.39 \mathrm{~m} . \mathrm{s}^{-1}\left(\mathrm{mlO}_{2} \cdot \mathrm{kg}^{-1} \cdot \mathrm{m}^{-1}\right)\end{array}$ & $0.110 \pm 0.02$ & $0.125 \pm 0.02$ & 0.044 & 0.023 \\
Six-minute walk distance $(\mathrm{m})$ & $665 \pm 59$ & $551 \pm 41$ & $<\mathbf{0 . 0 0 1}$ & 0.05 \\
\hline
\end{tabular}

592 Table 1. Comparisons between Achilles tendon stiffness, maximal plantarflexor strength, 593 energy cost of walking and six-minute walk distance between young and older adults. Values 594 are presented as mean $\pm \mathrm{SD} ; \alpha_{\mathrm{SID}}$ represents critical alpha level corrected for multiple 595 comparisons with Sidak-method.

596

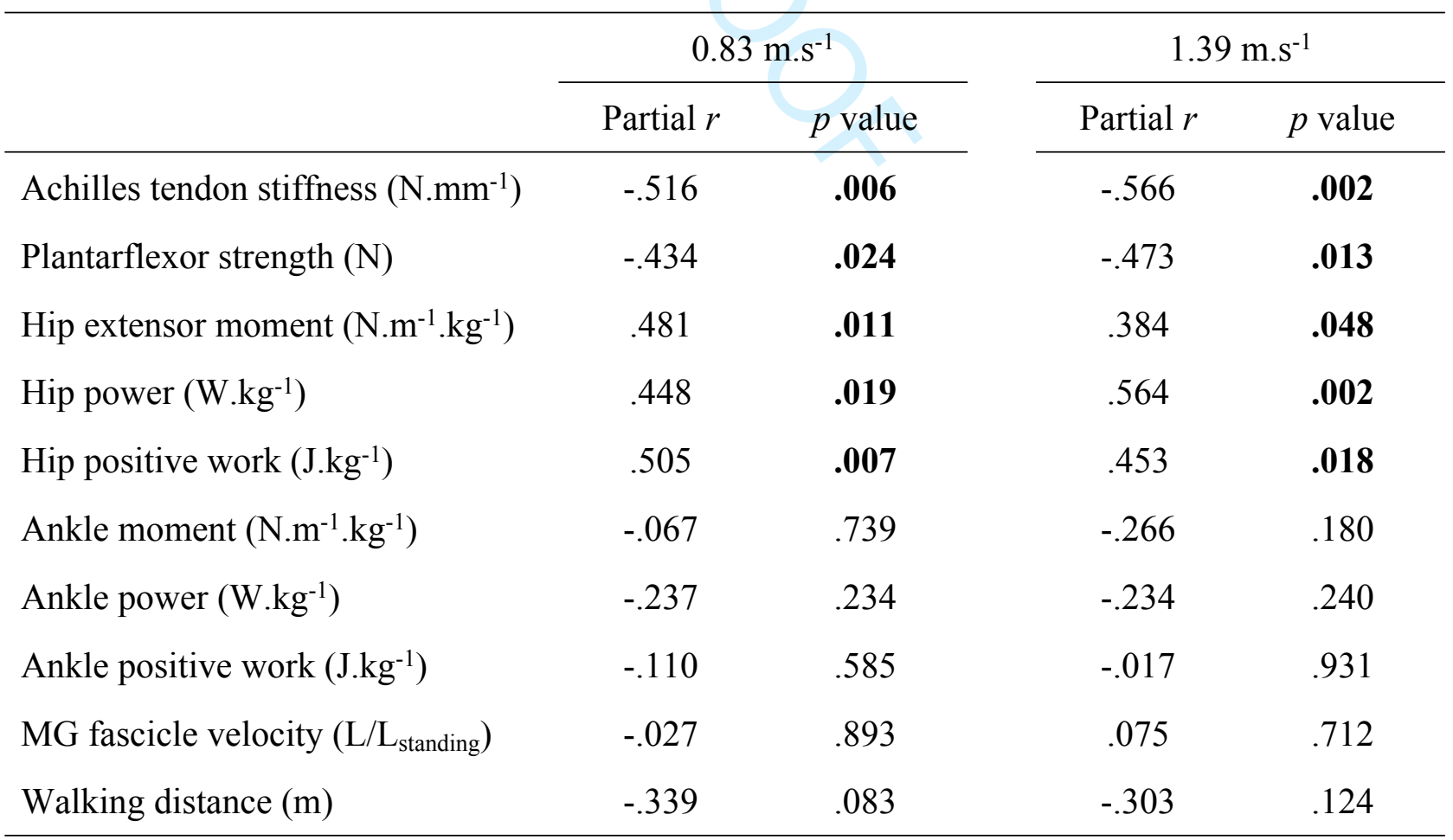

597 Table 2. Partial non-parametric correlations between independent variables and the energy cost 598 of walking at $0.83 \mathrm{~m} \cdot \mathrm{s}^{-1}$ and $1.39 \mathrm{~m} \cdot \mathrm{s}^{-1}$. Adjusted for step frequency. MG means medial 599 gastrocnemius. This value corresponds to the fascicle velocity at peak ankle moment during 600 stance. 


\begin{tabular}{|c|c|c|c|c|c|c|}
\hline & $R^{2}$ & $\begin{array}{l}\text { Adjusted } \\
\qquad R^{2}\end{array}$ & $\begin{array}{l}\text { Standard } \\
\text { error of } \\
\text { the } \\
\text { estimate }\end{array}$ & $\begin{array}{l}\text { Unstandardized } \\
\beta \text { coefficient }\end{array}$ & $\begin{array}{l}\text { Standardized } \\
\beta \text { coefficient }\end{array}$ & $\begin{array}{c}p \\
\text { value }\end{array}$ \\
\hline Model 1: $0.83 \mathrm{~m} . \mathrm{s}^{-1}$ & 0.448 & 0.379 & 0.015 & & & 0.002 \\
\hline (Constant) & & & & .093 & & \\
\hline Hip extensor moment & & & & .098 & .562 & 0.012 \\
\hline Plantarflexor strength & & & & $-4.50 \mathrm{E}^{-5}$ & -.159 & .426 \\
\hline Achilles tendon stiffness & & & & $-5.86 \mathrm{E}^{-6}$ & -.225 & .252 \\
\hline Model 2: 1.39 m.s $\mathrm{s}^{-1}$ & 0.418 & 0.345 & 0.018 & & & 0.004 \\
\hline (Constant) & & & & .027 & & \\
\hline Hip extensor moment & & & & .024 & .381 & 0.040 \\
\hline Plantarflexor strength & & & & .0001 & -.142 & .507 \\
\hline Achilles tendon stiffness & & & & .0001 & -.360 & .074 \\
\hline
\end{tabular}

601 Table 3. Adjusted multiple regression models at both slow and fast speeds with energy cost of 602 walking as dependent variable and plantarflexor strength, Achilles tendon stiffness and hip 603 extensor moment as independent variables. Hip extensors moment was the only significant 604 predictor of the models which predict between $35 \%$ and $38 \%$ of the variance. 
605

606

607

608

609

610

611

612

613

614

615

616

617

618

619

620

621

622

623

624

625

626

627

628

629

630

631

632

633

\section{$\underline{\text { Figure legends }}$}

Fig.1. Comparisons of medial gastrocnemius muscle-tendon length (A), tendinous tissue length (B), fascicle length (C) and fascicle velocity (D) during walking in young (blue lines) and older adults (red lines). Length were normalized respective to length measured during the static standing trial. Results for repeated measures ANOVA's with statistical non-parametrical mapping (SNPM) are depicted on the right (main effect group). No significant group differences were found at both speeds between young and older adults.

Fig.2. Comparisons of ankle moment (A) and hip moment (B) during walking in young (blue lines) and older (red lines) adults at $0.83 \mathrm{~m} \cdot \mathrm{s}^{-1}$ and $1.39 \mathrm{~m} . \mathrm{s}^{-1}$. Negative values indicate ankle plantarflexor moment and hip extensor moment. Results for repeated measures ANOVA's with statistical non-parametrical mapping (SNPM) are depicted on the right (main effect group). Larger peak ankle moment was found in young adults during the push-off phase. Larger hip extensor moment at early/mid-stance and hip flexor moment at pre-swing were observed in older adults.

Fig.3. Comparisons of ankle power (A) and hip power (B) during walking in young (blue lines) and older (red lines) adults at $0.83 \mathrm{~m} \cdot \mathrm{s}^{-1}$ and $1.39 \mathrm{~m} \cdot \mathrm{s}^{-1}$. Negative values indicate ankle plantarflexor moment and hip extensor moment. Results for repeated measures ANOVA's with statistical non-parametrical mapping (SNPM) are depicted on the right (main effect group). Larger peak ankle power was found in young adults during the push-off phase. Larger hip peak power was observed in older adults.

Fig.4. Ankle total positive work (A) and hip total positive work (B) during walking in young (blue circles) and older (red circles) adults at $0.83 \mathrm{~m} . \mathrm{s}^{-1}$ and $1.39 \mathrm{~m} . \mathrm{s}^{-1} . *$ indicates significant difference between young and older adults. Larger total positive work at the ankle was found in young adults at slow and fast speed. Inversely, larger total positive work at the hip was found in older adults at both speeds. 


\section{$0.83 \mathrm{~m} . \mathrm{s}^{-1} \quad 1.39 \mathrm{~m} . \mathrm{s}^{-1} \quad$ Main effect group}
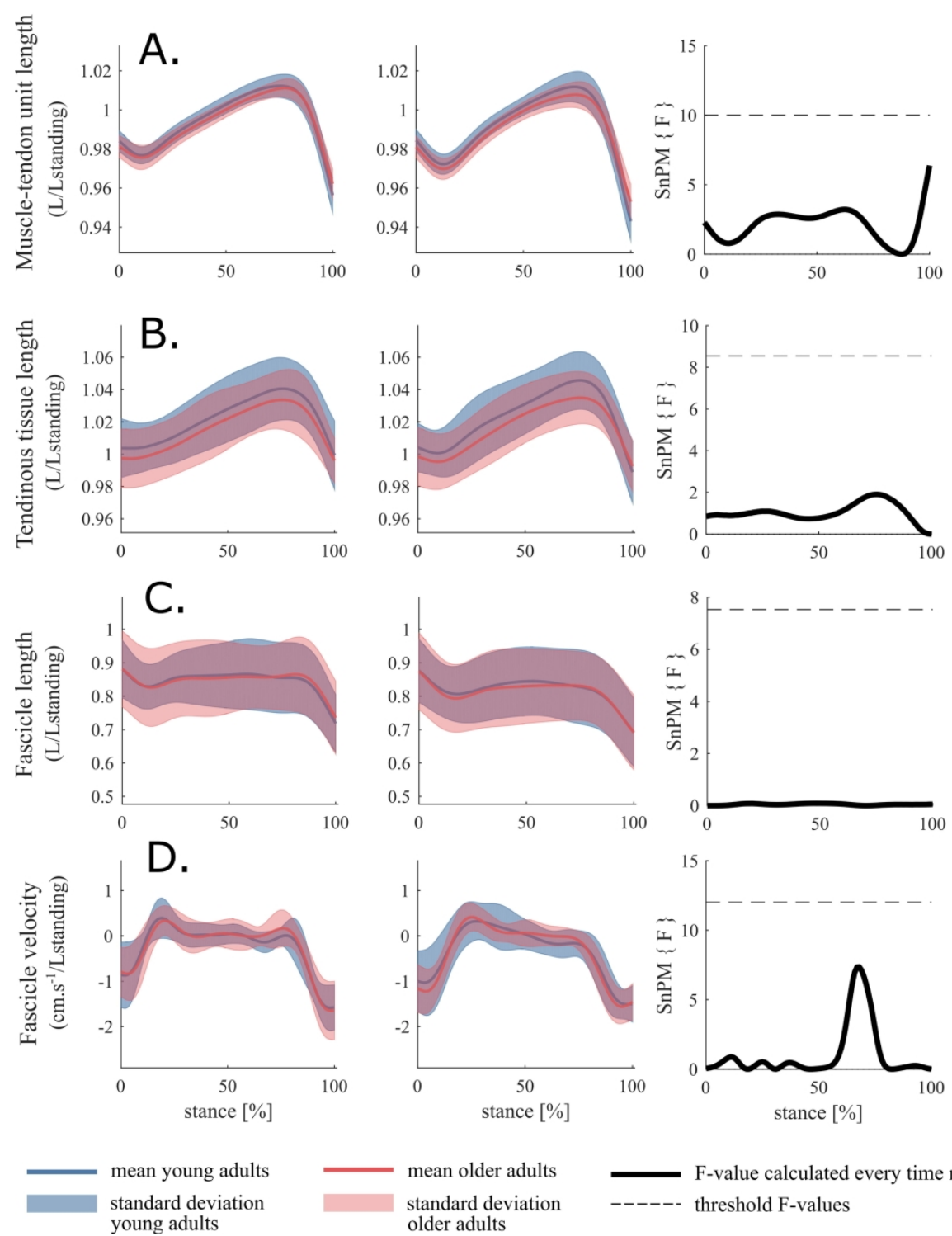

mean older adults standard deviation older adults

F-value calculated every time node young adults

\section{$211 \times 280 \mathrm{~mm}(300 \times 300$ DPI $)$}




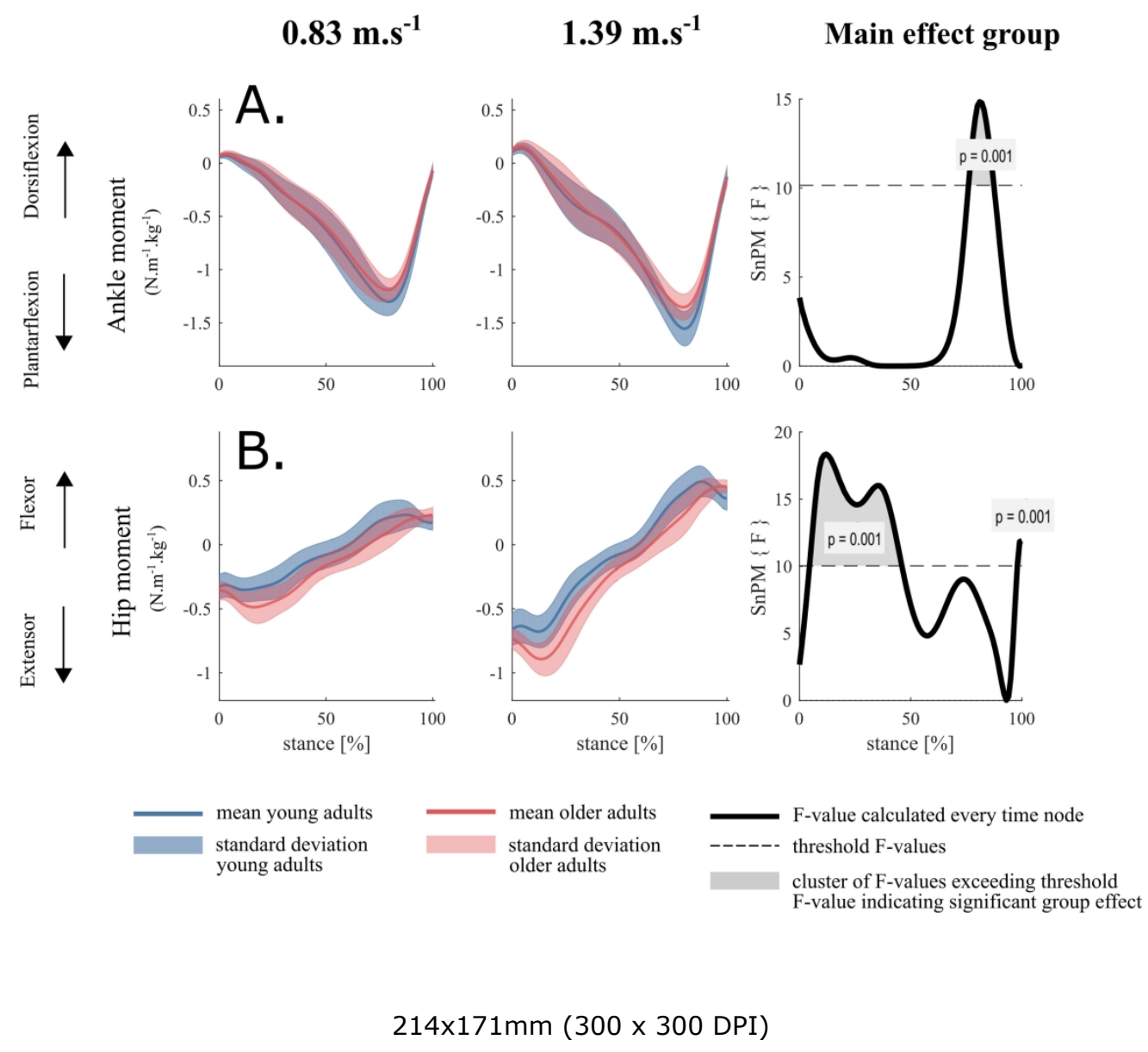

Scandinavian Journal of Medicine \& Science in Sports - PROOF 


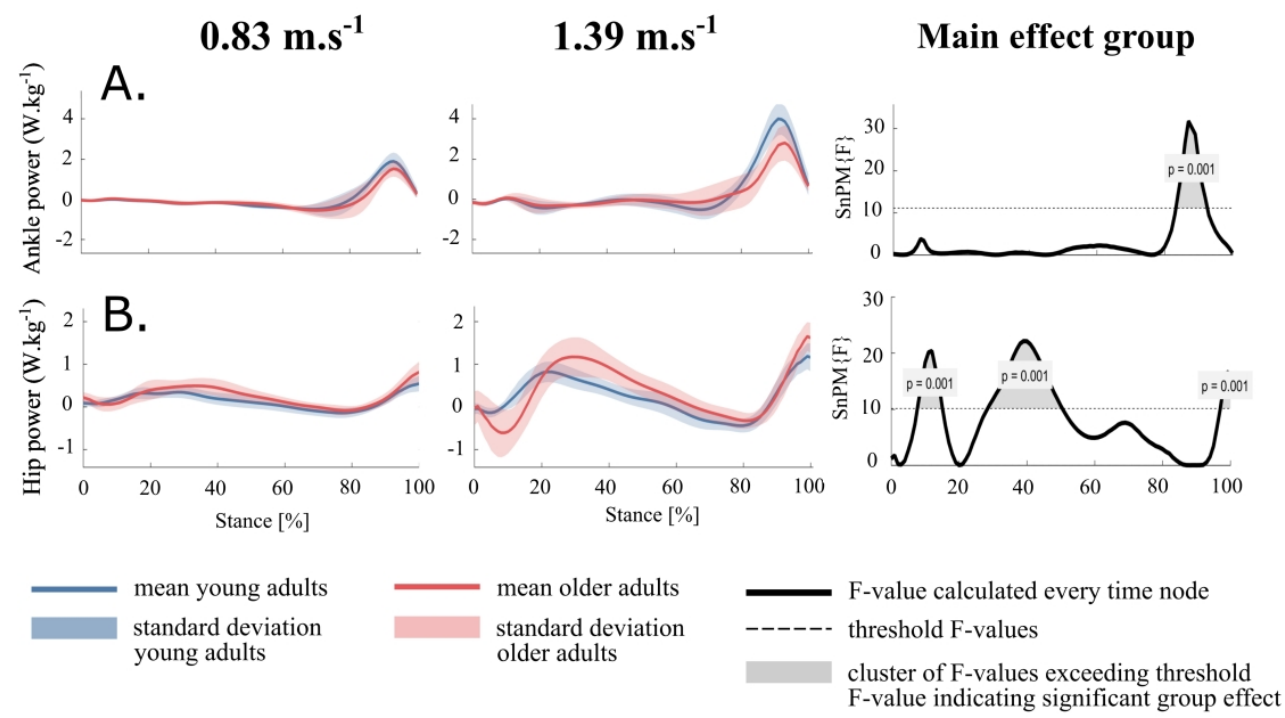




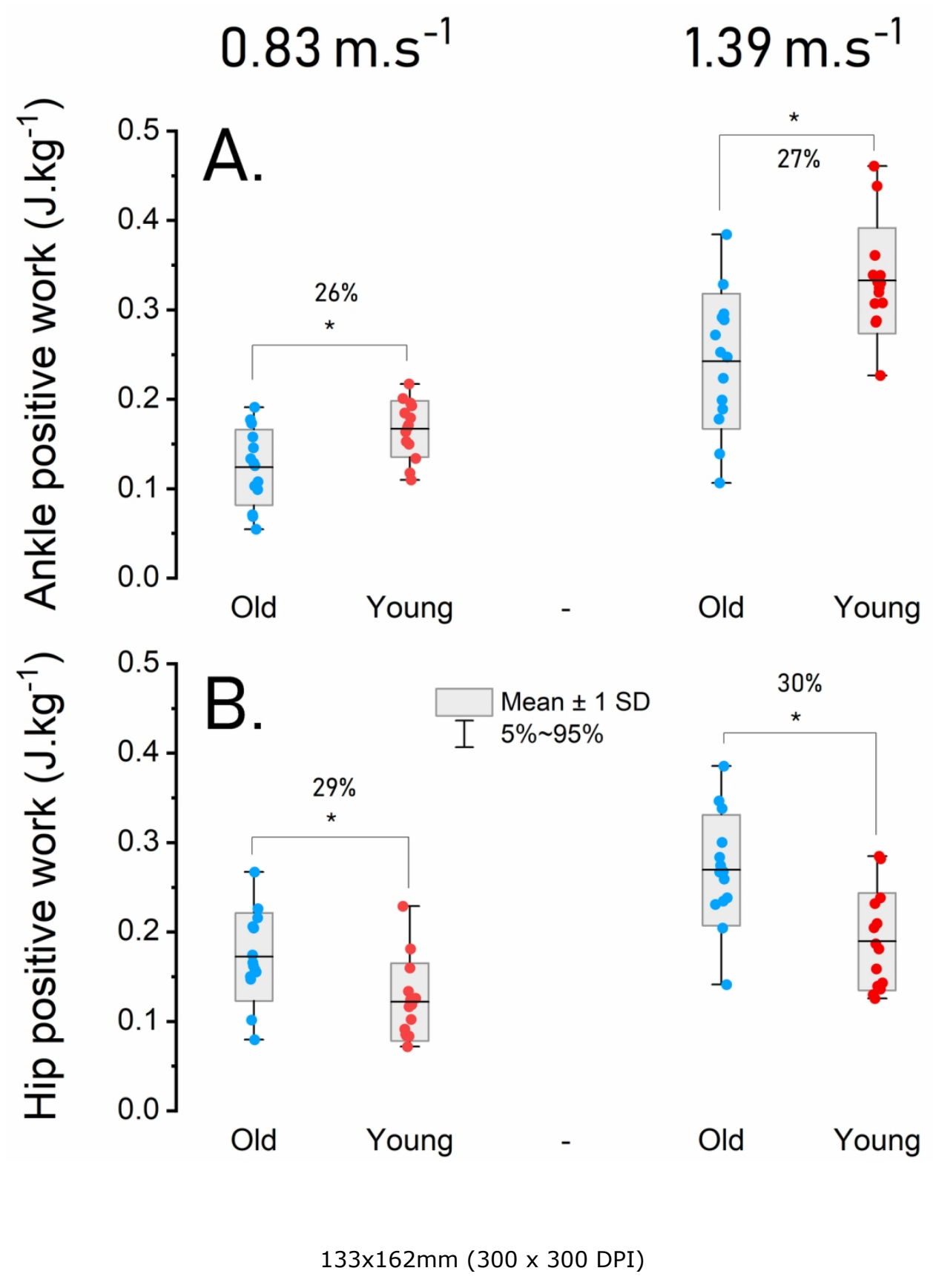

